\title{
Breast Cancer Surgery - a Forecast
}

\author{
Alberto Costa \\ European School of Oncology, Milan, Italy
}

The fact that breast cancer mortality is declining in most Western countries may give the impression that also the role of surgery may decline in the overall scenario of the control of this disease. Mortality is declining because of the increased awareness of the importance of breast cancer, because of the constant growth of early detection programs and because of the many improvements in treatment.

Surgery, however, sometimes seems to remain a bit in the corner, with most of the attention attracted to the different prevention/early-detection initiatives and to the overwhelming number of new drugs and biological compounds tested against the disease.

On the other hand, breast cancer surgeons have shown a nonnegligible commitment to progress in the control of the disease, first introducing the revolutionary concept of conservative surgery in the 1970s, then expanding the concept to the management of the axilla, then finding innovative solutions for operating non-palpable lesions, and recently adopting new techniques from plastic surgery and adapting them to oncological surgery (oncoplastic surgery).

Surgeons should be proud to have managed to considerably change their concepts in local and regional treatments of breast cancer, moving from the 'maximum tolerable treatment' of the 1970s to the 'minimum effective treatment' concept of the new millennium. Surgical research has continued to evaluate less radical procedures in order to minimize morbidity while maintaining local disease control, and confidence in this approach has increased.

The surgical management of non-palpable lesions has grown enormously in its importance, as a consequence of the dissemination of screening programs which bring to the attention of the surgeon a great number of new situations that require appropriate technology to be properly managed. A special credit should be given here to those investigators who had the idea of applying the sentinel node biopsy concept to the localiza- tion of non-palpable lesions (see the radioguided occult lesion localization (ROLL) procedure, i.e. the use of serum albumin macroaggregates, $10-150 \mu \mathrm{m}$ in diameter, labeled with Tc99 and injected into the lesion using stereotactic or ultrasound guidance) [1].

Together with the proper management of non-palpable lesions, breast surgeons have also to constantly cope with the issue of resection margins, because nothing is more frustrating for a surgeon than having to re-operate a patient for unsafe margins to find afterwards that there was no residual disease. The peritumoral spread of cancer cells is often so discontinuous that it is impossible to have a clear-cut and consistent attitude during the surgery. On the other hand, biopsies on the margin specimen are necessarily at random, which is why even a pathological diagnosis of 'negative margins' is never fully reassuring.

The near future should bring us some innovative concepts in this field, like the new probes that use radiofrequency spectroscopy to characterize tissues and that could significantly decrease re-excision rates in both palpable and non-palpable lesions [2], without leading to the excision of an excess of tissue volume.

Margin positivity is influenced by the extent of breast resection, and here is where special plastic surgery techniques could prove very useful. Well-performed subcutaneous mastectomies can give much better cosmetic results than conservative surgery in patients with small breasts or in disseminated ductal carcinoma in situ (DCIS). The improvement in surgical techniques that preserve also the nipple-areola complex will allow more and more to combine full radical excision of the disease together with excellent cosmetic results.

Another area where surgery might regain importance in the near future is the management of the internal mammary lymph nodes, which in selected cases could prove to be crucial in the proper definition of the prognosis and treatment plan-

\section{KARGER}

Fax +497614520714

Information@Karger.de

www.karger.com
(C) 2008 S. Karger GmbH, Freiburg

Accessible online at:

www.karger.com/brc
Prof. Alberto Costa, MD

European School of Oncology

Via del Bollo, 4

20123 Milan, Italy

Tel. +39 02 854645-26, Fax -45

director@eso.net 
ning, particularly in those cases in which both the axillary lymph nodes and the internal mammary ones are involved. Finally, very innovative scenarios are ahead of us as far as the interaction surgery-radiotherapy is concerned. No doubt that radiation therapy is clearly indicated after breast-conserving surgery. However, it is becoming more and more difficult for many patients to afford a 6-week treatment, particularly when they live far away from the radiotherapy center, or during the winter season when the daily journey to the hospital can become nearly more dangerous than a local recurrence of the disease (authentic comment by an elderly patient living in a Swiss mountain village at more than one-hour drive from the nearest radiotherapy center).

Great expectations are surrounding intraoperative radiotherapy (2009 could be the year of preliminary results of different ongoing clinical trials) and perioperative brachytherapy with disposable devices like the Mammosite balloon. There is an important role for the surgeon in all these innovative approaches, and it is not difficult to predict that better solutions for the patients will arise from a much closer surgeon-radiotherapist collaboration. Also nuclear medicine pretends to have a role to play in this field, and possibly more attention should be given to new methodologies currently under investigation like the intraoperative avidination for radionuclide treatment (IART) technology (injection of avidin by the surgeon into the tumor bed area, followed by $16-48 \mathrm{~h}$ postsurgery injection of intravenous $\left[{ }^{90} \mathrm{Y}\right]$ biotin) [3].
The real innovative approach, however, is the consequence of the fact that surgeons are becoming more and more aware of the incredibly multifaceted nature of breast cancer, with pathology reports now one or two pages long and full of extremely detailed information like grading, proliferation index, vascular invasion, HER2-neu expression on top of the traditional size of the tumor, histotype, lymph node and hormone receptor status. There are at least a couple of hundreds of possible combinations of these factors and parameters, making the biology of breast cancer more and more complex. Gene profiling and research on stem cells will do the rest and very soon there will be no room for surgeons still thinking that the more you cut, the more you cure. Good for breast cancer surgery, and for breast cancer patients.

\section{References}

1 Intra M, de Cicco C, Gentilini O, Luini A, Paganelli G: Radioguided localisation (ROLL) of non-palpable breast lesions and simultaneous sentinel lymph node biopsy (SNOLL): the experience of the European Institute of Oncology. Eur J Nucl Med Mol Imaging 2007;34:957-958.

2 Allweis TM, Kaufman Z, Lelcuk S, et al.: A prospective, randomized, controlled, multicenter study of a real-time, intraoperative probe for positive margin detection in breast-conserving surgery. Am J Surg 2008;196:483-489.

3 Paganelli G, Ferrari M, Cremonesi M, et al.: IART: Intraoperative avidination for radionuclide treatment. A new way of partial breast irradiation. Breast 2007;16: $17-26$. 